\title{
Can We Improve Thyroid Fine-needle Aspiration Cytology Adequacy in a Low-volume Thyroid Center? \\ Results of Surgeon-performed Ultrasound-guided Fine-needle Aspiration Cytology
}

\author{
Mohamed Shaaban, Mario Metry, and Sebastian Aspinall \\ Endocrine and Breast Surgery Unit, Northumbria Healthcare NHS Foundation Trust, Ashington, UK
}

DOI: https://doi.org/10.17925/OHR.2017.13.01.21

\begin{abstract}
ntroduction: Thyroid cancer is the most common endocrine malignancy, and has shown an increase in incidence in recent decades. Fine-needle aspiration cytology (FNAC) is the mainstay of assessment of thyroid nodules and diagnosis of malignancy. Several reports have suggested that ultrasound (US)-guided FNAC has many advantages over palpation-guided biopsy. Methods: Comparison of results of thyroid nodule FNACs in a low volume thyroid center (Northumbria Health Care NHS Foundation Trust) performed by a diverse group of clinicians and radiologists involved in the management of thyroid nodules over 18 months, between October 2008 and April 2010, identified retrospectively from pathology records, with the results of surgeon-performed US (SUS) -guided FNACs performed by a single operator (SRA) recorded prospectively over 17 months between July 2013 and November 2014. Results: The study included 185 FNA, with 104 FNAC being undertaken between October 2008 and April 2010 compared to 81 FNAC between July 2013 and November 2014. There was a statistically significant reduction of non-diagnostic rates in the second period: 23/81 (28\%) versus 51/104 (49\%) (Fisher's exact, p=0.0063), non-neoplastic (Thy2) scores remained comparable: 28/81 (35\%) versus 32/104 (31\%), there were more Thy3 results: 21/81 (26\%) versus 15/104 (14\%), while the proportion of neoplastic results in the SUS-FNAC group increased: 8/81 (10\%) versus 6/104 (6\%). Also, time to diagnostic FNAC was significantly shorter in the SUS-FNAC group: $24.2 \pm 4.5$ versus $54.9 \pm 11.4$ days ( $p=0.01$, unpaired t-test). Conclusion: SUS-guided FNAC for thyroid nodules is a safe and simple technique. This study demonstrates that it leads to improved patient care by reducing inadequacy rate and time to diagnosis in a low-volume thyroid center.
\end{abstract}

\section{Keywords}

Thyroid nodule, surgeon-performed ultrasonography, fine-needle aspiration cytology (FNAC), adequacy

Disclosure: Mohamed Shaaban, Mario Metry, and Sebastian Aspinall have no conflicts of interest to declare. No funding was received in the publication of this article. Approva was obtained to carry out this audit, verbal consent was obtained for each fine-needle aspiration procedure.

Compliance with Ethics: All procedures were followed in accordance with the responsible committee on human experimentation and with the Helsinki Declaration of 1975 and subsequent revisions.

Authorship: All named authors meet the International Committee of Medical Journal Editors (ICMJE) criteria for authorship of this manuscript, take responsibility for the integrity of the work as a whole, and have given final approval to the version to be published.

Open Access: This article is published under the Creative Commons Attribution Noncommercial License, which permits any noncommercial use, distribution, adaptation, and reproduction provided the original author(s) and source are given appropriate credit.

Received: November 6, 2016

Accepted: January 3, 2017

Citation: Oncology \& Hematology Review, 2017;13(1):21-4

Corresponding Author: Mohamed Shaaban, Endocrine and Breast Surgery Unit, Northumbria Healthcare NHS Foundation Trust, Woodhorn Lane, Ashington NE63 9JJ, UK E: dr.mohamed.shaaban@gmail.com
Thyroid cancer is the most common endocrine malignancy, and has increased in incidence in recent decades. ${ }^{1-3}$ Fine-needle aspiration cytology (FNAC) is the mainstay of assessment of thyroid nodules and diagnosis of malignancy. ${ }^{4,5}$ Several reports have suggested that ultrasound (US)-guided FNAC has many advantages over palpation-guided biopsy. It is now generally recommended that thyroid nodules should be biopsied under US-guidance to improve diagnostic accuracy (British Thyroid Association Guidelines for the Management of Thyroid Cancer 2014). ${ }^{6-8}$

Low adequacy rates of FNAC for thyroid nodules remains an issue, particularly in low-volume thyroid units with multiple clinicians performing this investigation. This leads to repeated FNACs and delays in diagnosis. Several strategies have been used to try to improve the diagnostic yield. ${ }^{9}$

It was apparent that insufficiency rates of thyroid FNACs were high in Northumbria Trust, leading to increased patient hospital attendance, anxiety, delay in diagnosis and treatment. These audits were undertaken to assess whether surgeon-performed US (SUS)-guided FNAC could improve the adequacy rates of thyroid nodules FNAC and shorten the time to diagnosis in a low-volume thyroid center.

\section{Methods}

In the first period, the pathology department provided us with a list of all thyroids FNACs performed in the trust by a diverse group of clinicians 
Table 1: Comparison of the thyroid nodule fine-needle aspiration cytology (FNAC) from all clinicians using a variety of techniques and surgeon-performed ultrasound-guided FNAC

\begin{tabular}{|l|l|l|}
\hline FNAC score & First audit & Second audit \\
\hline Thy1 (non-diagnostic) & $51(49 \%)$ & $23(28 \%)$ \\
\hline Thy2 (non-neoplastic) & $32(31 \%)$ & $28(35 \%)$ \\
\hline Thy3 (indeterminate) & $15(14 \%)$ & $21(26 \%)$ \\
\hline Thy4/5 (suspicious of malignancy/malignant) & $6(6 \%)$ & $8(10 \%)$ \\
\hline Total & $\mathbf{1 0 4}$ & $\mathbf{8 1}$ \\
\hline
\end{tabular}

Figure 1: Time to diagnosis statistics chart

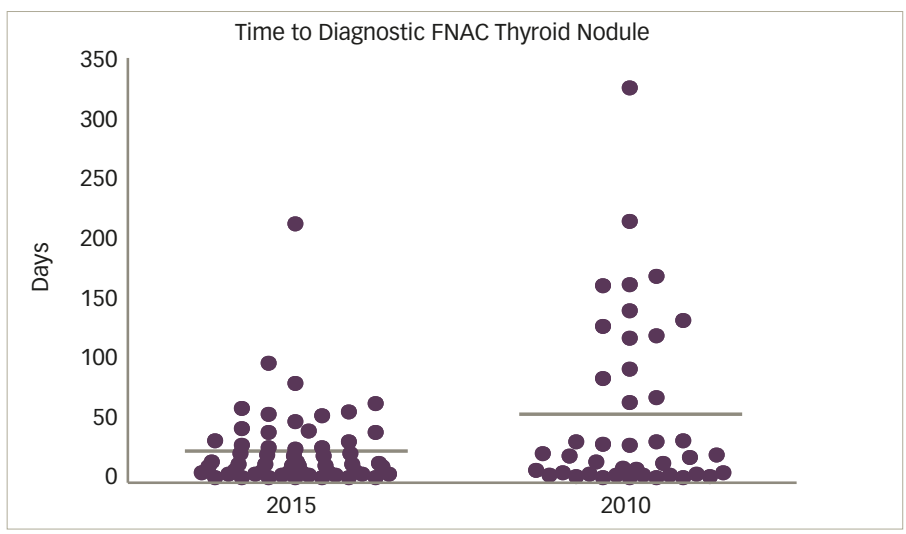

FNAC = fine-needle aspiration cytology

and radiologists from October 2008 to April 2010, and we retrospectively reviewed the case notes and clinic letters to ascertain other clinical information. The second period included only those FNACs carried out by a single surgeon (SRA) under US-guidance from July 2013 to November 2014 and data were recorded prospectively.

In the first period, a diverse group of FNAC techniques were performed either free hand or under US-guidance, using needles of different calibres, with or without suction according to the preference of the clinician. In the second period a standard technique was employed using a Sonosite Edge ${ }^{\text {TM }}$ US system (FUJIFILM SonoSite, Inc., Washington, US) with 13-6 MHz transducer. FNAC was obtained using $21 \mathrm{G}$ needle without suction, inserted into the nodule under US-guidance with rotation and multiple passes (>10). Aspirates were expelled and smeared onto glass slides and air dried or fixed in alcohol prior to cytopathology analysis according to Royal College of Pathologists guidelines. ${ }^{10}$

Cytopathology analysis was undertaken by several pathologists in Northumbria Healthcare NHS Foundation Trust (NHCT). Statistical analysis was carried with GraphPad Prism ${ }^{\text {TM }}$ (GraphPad Software, Inc., California, US) and $p<0.05$ taken as significant.

\section{Results}

From 85 patients, 104 FNACs were identified in the first period and 81 FNACs from 49 patients were identified in the second. For nondiagnostic FNAC (Thy1) there was a statistically significant reduction of non-diagnostic FNAC rates in the second period: 23/81 (28\%) versus 51/104 (49\%) in the SUS-FNAC group (Fisher's exact, $p=0.0063$ ). For non-neoplastic FNAC (Thy2), scores remained comparable: 28/81 (35\%) versus 32/104 (31\%). For indeterminate FNAC (Thy3), there were more Thy3 results: $21 / 81$ (26\%) versus $15 / 104(14 \%)$ in the SUS-FNAC group. However, this difference just failed to reach statistical significance (Fisher's exact, $p=0.0615$ ). For suspected malignancy/malignant FNAC (Thy4/5), while the proportion of Thy4 and Thy 5 results in the SUS-FNAC group increased: $8 / 81$ (10\%) versus 6/104 (6\%), overall numbers of suspicious or malignant FNAC remained low. Time to diagnostic FNAC - defined as time from decision to perform first FNAC to diagnostic (Thy2 or above) FNAC report-was significantly shorter in the SUS-FNAC group $24.2 \pm 4.5$ versus $54.9 \pm 11.4$ days $(p=0.01$, unpaired t-test) (see Figure 1).

\section{Discussion}

FNAC is a simple and safe procedure that has reduced the proportion of patients undergoing unnecessary thyroidectomy, while serious adverse events are rare. This was illustrated in a systematic review of 13 studies including 18,156 individuals undergoing thyroid biopsy. ${ }^{11}$ When taking a FNAC, it is important to avoid a bloody specimen as blood may obscure the thyroid epithelial cells and make cytological interpretation difficult.

Ideally, the overall accuracy of FNAC should exceed $95 \%$, with a falsenegative rate for a benign interpretation between 0 and $3 \% .{ }^{9.12}$ Repeat FNAC reduces the false-negative rate and in one series patients with benign cytology had a $90 \%$ probability of benign disease, when confirmed with repeat FNAC, this probability increased to $98 \%$.12,13

The positive predictive value of a malignant cytology is $97-99 \%$, as was shown in a report of 3,589 nodules from 2,587 patients evaluated and aspirated under thyroid US-guidance..$^{14}$ In this study, the predictive value of a malignant versus non-malignant cytological diagnosis was $97 \%$ and $99.7 \%$, respectively. In a similar report of 4,703 FNAC samples, the sensitivity and specificity of thyroid FNAC for the diagnosis of malignancy were $94 \%$ and $98.5 \%$, respectively. ${ }^{15}$ Hence, the accuracy and clinical utility of thyroid FNAC is excellent. However, Janczak et al. found that, based on 108 thyroid cancers resected with 66 FNA during a 20-year period, only 14 FNA biopsies (21\%) revealed cancer, all of which were confirmed in the postoperative specimen, although six cases of FNA-diagnosed cancer revealed a different histological type postoperatively. ${ }^{16}$

The non-diagnostic FNAC (Thy1) rate varies from 3\% to $10 \%$ in experienced centers. The absence of malignant thyroid epithelial cells should not be interpreted as an absence of malignancy in the nodule if there are insufficient thyroid epithelial cells present in the aspirate. The Royal college of Pathologists" guidelines recommend that "there should be at least six groups of thyroid follicular epithelial cells across the slides containing at least 10 well-visualized thyroid epithelial cells" ${ }^{10}$ If repeated inadequate FNACS are obtained, it is recommended to repeat the FNAC under USguidance according to the American Thyroid Association (ATA) guidelines." A cutting needle (core) biopsy under US-guidance may also help if repeated FNAC remain insufficient for diagnosis. ${ }^{17}$

In contrast, other studies found higher insufficiency rates of approximately $15-20 \%$ for percutaneous thyroid FNACs. The highest frequency of nondiagnostic cytology occurs in cystic nodules and in very small nonpalpable nodules. ${ }^{18,19}$ The diagnostic yield of sampling palpable nodules is 
Figure 2: Findings of 2011 audit

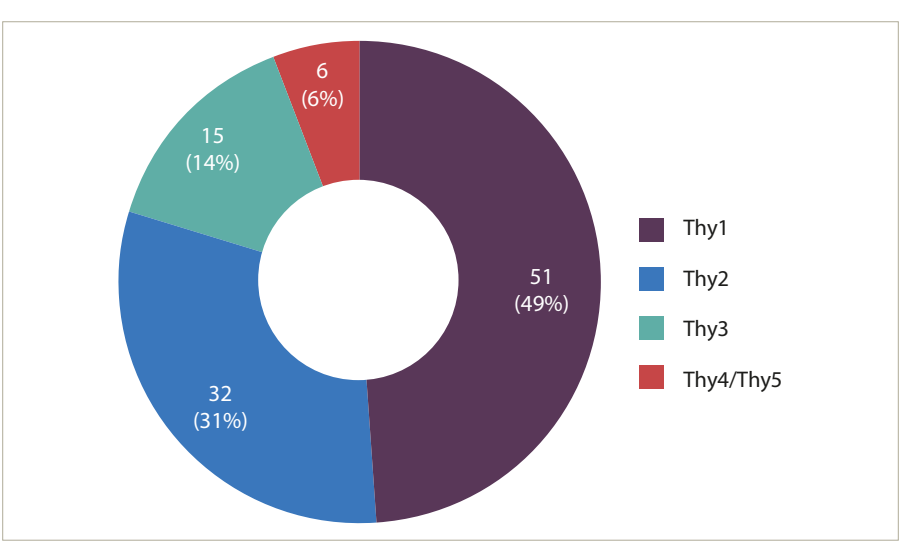

FNAC = Fine-needle aspiration cytology; Thy1 = nondiagnostic FNAC;

Thy 2 = nonneoplastic FNAC; Thy 3 = indeterminate FNAC; Thy $4 / 5=$ suspected

malignancy/malignant FNAC.

enhanced when US-guidance is employed, especially when a nodule has undergone cystic deterioration. If initial US-guided FNA is non-diagnostic, a repeat US-guided FNA will yield a diagnostic cytology specimen in 75\% of solid and $50 \%$ of cystic nodules..$^{18}$ If there is very sparse or no aspirated material, using a larger lumen needle may result in a satisfactory specimen. ${ }^{17}$ US-guidance is preferred to palpation-guided FNAC, as the use of US improves the cytologic diagnostic accuracy rate and reduces the non-diagnostic rate..$^{20-23}$

In low volume thyroid centers, due to the small number of thyroid nodule FNACs, it is impractical to have instant cytological reporting of thyroid FNACs to assess for adequacy of follicular epithelial cell numbers, due to the low numbers of cases seen, though this has shown to be a useful adjunct to FNAC to minimize inadequacy rates in high-volume centers. ${ }^{24}$ Concentrating the workload into the hands of dedicated, interested clinicians and pathologists may improve the outcome of FNAC. ${ }^{25,26}$ Multiple authors are now in favor of surgeon-performed US-guided FNACS as this has proved to provide many advantages, especially in shortening the time to diagnosis and expediting management, ${ }^{27,28}$ which is in line with our findings in this study.

Regarding the technique, samples of thyroid tissue may be obtained percutaneously with a narrower-gauge needle than that used for SUSguided FNAC, such as 25 or 27 gauge, to obtain cells for cytological analysis. Alternatively, some clinicians prefer to use a cutting needle to obtain a core of tissue for histopathological study. It should be noted, however, that there may be more complications, such as hematoma, when a core specimen is obtained. ${ }^{29}$ FNAC is therefore preferred to core needle biopsies at most centers. The fine-needle technique is popular because it is low risk and cytology provides diagnostic information about nuclear and cytoplasmic detail that is not available with a core biopsy..$^{30}$

The technique for US-guided fine-needle thyroid biopsy involves three steps: puncture of the nodule or goiter, obtaining the sample, and preparation of the slides. With the surgeon performing the biopsy, results
Figure 3: Findings of 2015 audit

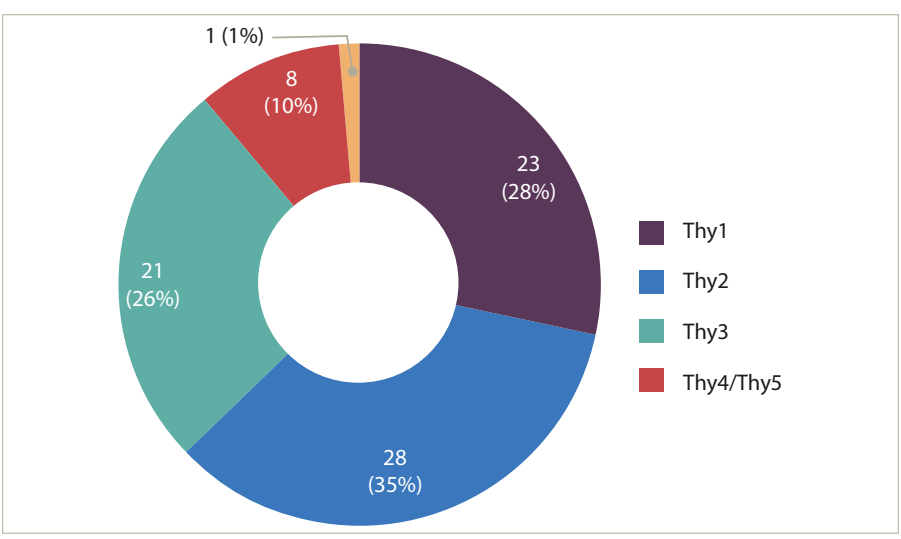

FNAC = Fine-needle aspiration cytology; Thy1 = nondiagnostic FNAC; Thy 2 = nonneoplastic FNAC; Thy $3=$ indeterminate FNAC; Thy $4 / 5=$ suspected malignancy/malignant FNAC.

from some studies showed that it is technically feasible, ${ }^{22}$ increases adequacy and efficacy, especially with the presence of immediate cytopathology assessment, ${ }^{24,26}$ and shortens time for diagnosis of thyroid nodules, which has implications for oncological benefit as well. ${ }^{28} \mathrm{Hilmi}$ Bozkurt et al. reported that US-guided FNAC can be performed with a low non-diagnostic rate as experience grows. ${ }^{31}$

The following limitations were inherent in this study and may have biased the results. The first audit cycle demonstrated a very high rate of insufficiency in thyroid FNAC (higher than that reported in the literature) (see Figure 2). We think the reason our non-diagnosis rate was so high in the first period was that multiple clinicians were involved in performing a small number of thyroid FNAC, with or without SUS, in a low-volume thyroid center, which seems to lead to high non-diagnosis rate. Concentrating thyroid FNAC in the hands of dedicated, interested clinicians who audit their results seems to reduce our non-diagnosis rate and time to diagnosis. Although the second period did significantly improve the adequacy of FNAC, and shortened time to diagnosis, the inadequacy rate remained high (28\%), suggesting that there is still considerable room for improvement (see Figure 3). However, there were clear benefits to service delivery from the use of SUS FNAC. Results from the first period were collected retrospectively and so are inherently less accurate than the prospectively collected data from the second cycle. Thyroid nodules in the second period were referred to a surgeon for assessment, so may represent a different spectrum of pathology to those assessed by all clinicians in the first period. The surgeon may have sent potentially difficult cases to radiology for FNAC in the second period, thus biasing the results. The first period also included FNACs taken free hand, which has been shown to be inferior to US-guided FNAC.

\section{Conclusion}

SUS-guided FNAC for thyroid nodules is a safe and simple technique. This study demonstrates that it leads to improved patient care by reducing inadequacy rate and shortens time to diagnosis in a low-volume thyroid center. $\square$ 
1. Burgess $J R$, Tucker $P$, Incidence trends for papillary thyroid carcinoma and their correlation with thyroid surgery and thyroid fine-needle aspirate cytology, Thyroid, 2006;16:47-53.

2. Davies $\mathrm{L}$, Welch $\mathrm{HG}$, Increasing incidence of thyroid cancer in the United States 1973-2002, JAMA, 2002;295:2164-7.

3. Leenhardt $L$, Grosclaude $P$, Cherie-Challine $L$, et al., Increased incidence of thyroid carcinoma in France: a true epidemic or thyroid nodule management effects? France: Report from the French Thyroid Cancer Committee, Thyroid, 2004;14:1056-60.

4. Cooper DS, Doherty GM, Haugen BR, et al., Management guidelines for patients with thyroid nodules and differentiated thyroid cancer, Thyroid, 2006:16:109-42.

5. Yang GC, Liebeskind D, Messina AV, Ultrasound guided fine-needle aspiration of the thyroid assessed by ultrafast Papanicolaou stain; data from 1135 biopsies with a two to six-year follow-up, Thyroid, 2001;11:581-9.

6. Cesur M, Corapcioglu D, Bulut S, et al., Comparison of palpationguided fine-needle aspiration biopsy to ultrasound-guided fine-needle aspiration biopsy in the evaluation of thyroid nodules, Thyroid, 2006;16:555-61.

7. Cai XJ, Valiyaparambath N, Nixon P, et al., Ultrasound-guided fine needle aspiration cytology in the diagnosis and management of thyroid nodules, Cytopathology, 2006;17:251-6.

8. Perros P, Boelaert K, Colley S, et al.,Guidelines for the management of thyroid cancer, Clin Endocrinol, 2014;81(Suppl 1):1-122.

9. Yassa $\mathrm{L}$, Cibas $\mathrm{ES}$, Benson $\mathrm{CB}$, et al., Long-term assessment of a multidisciplinary approach to thyroid nodule diagnostic of a multidisciplinary approach to thy

10. The Royal College of Pathologists, Guidance on the reporting of thyroid cytology specimens, London: The Royal College of Pathologists, 2016;G089. Available at: www.rcpath.org/ asset/7D693CE4-0091-4621-97F79E2A0D 1034D6/ (accessed 26 February 2017).

11. Polyzos SA, Anastasilakis AD, Clinical complications following thyroid fine-needle biopsy: a systematic review, Clin Endocrino (Oxf), 2009;71:157-65.

12. Cibas ES, Ali SZ, The Bethesda System for Reporting Thyroid Cytopathology, Thyroid, 2009;19:1159-65.

13. Oertel YC, Miyahara-Felipe L, Mendoza MG, Yu K, Value of repeated fine needle aspirations of the thyroid: an analysis of over ten thousand FNAS, Thyroid, 2007;17:1061-66.

14. Polyzos $S A$, Anastasilakis $A D$, Systematic review of cases reporting blood extravasation-related complications after thyroid fine-needle biopsy, J Otolaryngol Head Neck Surg, 2010;39:531-41.

15. Yang J, Schnadig V, Logrono R, Wasserman PG, Fine-needle aspiration of thyroid nodules: a study of 4703 patients with histologic and clinical correlations, Cancer, 2007;111:306-15.

16. Janczak D, Pawlowski W, Dorobisz T, et al., An evaluation of the diagnostic efficacy of fine needle aspiration biopsy in patients operated for a thyroid nodular goitre, OncoTargets Ther, 2016;9:5819-23.

17. Haugen BR, Alexander EK, Bible KC, et al., The American Thyroid Association Guidelines Task Force on Thyroid Nodules and Diff. USA: American Thyroid Association Management Guidelines for Adu. American Thyroid Association Management Guidelines for Cancer, Thyroid, 2015;26:1

18. Alexander EK, Heering JP, Benson CB, Assessment of nondiagnostic ultrasound-guided fine needle aspirations of thyroid nodules, I Clin Endocrinol Metab, 2002;87:4924-7.

19. Mittendorf EA, Tamarkin SW, MCHenry CR, The results of ultrasound-guided fine-needle aspiration biopsy for evaluation of nodular thyroid disease, Surgery, 2002;132:648-53.

20. Tollin SR, Mery GM, Jelveh N, et al., The use of fine-needle aspiration biopsy under ultrasound guidance to assess the risk of malignancy in patients with a multinodular goiter, Thyroid 2000;10:235-41.

21. Leenhardt L, Hejblum G, Franc B, et al., Indications and limits of ultrasound-guided cytology in the management of nonpalpable thyroid nodules, J Clin Endocrinol Metab, 1999;84:24-28.
22. Danese D, Sciacchitano S, Farsetti A, et al., Diagnostic accuracy of conventional versus sonography-guided fine-needle aspiration biopsy of thyroid nodules, Thyroid, 1998;8:15-21.

23. Carmeci C, Jeffrey RB, MCDougall IR, et al., Ultrasound-guided fine-needle aspiration biopsy of thyroid masses, Thyroid, 1998;8:283-9.

24. O'Malley ME, Weir MM, Hahn PF, et al., US-guided fine-needle aspiration biopsy of thyroid nodules: adequacy of cytologic material and procedure time with and without immediate cytologic analysis, Radiology, 2002;222:383-7.

25. Ahn D, Sohn JH, Yeo CK, Jeon JH, Feasibility of surgeon-performed ultrasound-guided core needle biopsy in the thyroid and lymph nodes, Head Neck, 2016;38 (Suppl 1):E1413-8.

26. Witt RL, Sukumar VR, Gerges F, Surgeon-performed ultrasoundguided FNAC with on-site cytopathology improves adequacy and accuracy, Laryngoscope, 2015;125:1633-6.

27. Ahn D, Kim H, Sohn JH, et al., Surgeon-performed ultrasoundguided fine-needle aspiration cytology of head and neck mass lesions: sampling adequacy and diagnostic accuracy, Ann surg Oncol, 2015;22:1360-5

28. Gu WX, Tan CS, Ho TW, et al., Surgeon-Performed UltrasoundGuided Fine-Needle Aspiration Cytology (SP-US-FNAC) Shortens Time for Diagnosis of Thyroid Nodules, Ann Acad Med Singapore 2014;43:320-4.

29. Kakiuchi Y, Idota N, Nakamura M, Ikegaya H, A Fatal Case of Cervical Hemorrhage After Fine Needle Aspiration and Core Needle Biopsy of the Thyroid Gland, Am J Forensic Med Pathol, 2015;36:207-9.

30. Harvey JN, Parker D, De P, et al., Sonographically guided core biopsy in the assessment of thyroid nodules, $J$ Clin Ultrasound 2005;33:57-62.

31. Bozkurt H, Irkörücü O, Aziret M, et al., Comparison of 1869 thyroid ultrasound-guided fine-needle aspiration biopsies between general surgeons and interventional radiologists, Ann Med Surg (Lond), 2016;10:92-102. 